\title{
Analytic Formulation and Numerical Implementation of an Acoustic Pressure Gradient Prediction
}

\author{
Seongkyu Lee* and Kenneth S. Brentner \\ Department of Aerospace Engineering \\ The Pennsylvania State University \\ University Park, Pennsylvania, U.S.A. \\ F. Farassat ${ }^{\ddagger}$ \\ NASA Langley Research Center \\ Hampton, Virginia, U.S.A.
}

\begin{abstract}
The scattering of rotor noise is an area that has received little attention over the years, yet the limited work that has been done has shown that both the directivity and intensity of the acoustic field may be significantly modified by the presence of scattering bodies. One of the inputs needed to compute the scattered acoustic field is the acoustic pressure gradient on a scattering surface. Two new analytical formulations of the acoustic pressure gradient have been developed and implemented in the PSU-WOPWOP rotor noise prediction code. These formulations are presented in this paper. The first formulation is derived by taking the gradient of Farassat's retarded-time Formulation 1A. Although this formulation is relatively simple, it requires numerical time differentiation of the acoustic integrals. In the second formulation, the time differentiation is taken inside the integrals analytically. The acoustic pressure gradient predicted by these new formulations is validated through comparison with the acoustic pressure gradient determined by a purely numerical approach for two model rotors. The agreement between analytic formulations and numerical method is excellent for both stationary and moving observers case.
\end{abstract}

*Graduate Research Assistant; sxl348@psu.edu

†Associate Professor; Associate Fellow AIAA; ksbrentner@psu.edu

${ }^{\ddagger}$ Senior Research Scientist; Fellow AIAA; feri.farassat@nasa.gov 


\section{Introduction}

Acoustic scattering of the noise generated by rotating blades is an area of research that is not well developed. For example, a helicopter fuselage, a tiltrotor wing, or the duct surrounding a fan, each may substantially modify the acoustic signal that arrives at an arbitrary observer location. Such a modification would change both the magnitude and directivity of the acoustic signal from what would be observed for an isolated rotor. The significant effect of fuselage on the noise field generated by a rotating point source was demonstrated by Atalla and Glegg ${ }^{1,2}$ using a ray-acoustics approach.

Tools exist for predicting fan noise scattering in turbofan engines, but only limited work has been done on the acoustic scattering of rotor noise by short ducts (i.e., ducted tail rotors; ducted propellers for compound rotorcraft; ducted fans in UAVs, etc.) The various numerical approaches $^{3-5}$ to solve the acoustic scattering problem use acoustic velocity on a scattering surface as a boundary condition. For example, a rigid surface implies either the satisfaction of the impenetrability condition on the surface or zero normal acoustic velocity relative to the scattering surface. Most conventional acoustic codes compute acoustic pressure at an observer, not the acoustic velocity, but the gradient of the acoustic pressure is connected with the acoustic velocity through the linearized momentum equation. In other words, the calculation of the acoustic pressure gradient on a scattering surface is required to fulfill the boundary condition for the scattering problem. The calculation of the acoustic pressure gradient is, therefore, a key aspect in solving the acoustic scattering problem.

A numerical evaluation of the pressure gradient, which requires evaluation of the spatial derivative of acoustic pressure with respect to each direction, is the simplest way to calculate the pressure gradient on the surface. Nevertheless, it is computationally expensive. Therefore, it is not practical to calculate the pressure gradient numerically for a realistic helicopter configuration, where the scattering computation may require the acoustic pressure gradient at thousands or even tens of thousands of collocation points on the scattering surface. It is important to develop computationally efficient analytic formulations for the pressure gradient to enable routine acoustic scattering predictions.

\section{Research Objective}

Rotor noise is well described by Farassat's formulation 1A, which is an integral representation of the solution of the Ffowcs Williams-Hawkings (FW-H )equation. ${ }^{6}$ With this formulation, the accurate prediction of rotor noise largely depends upon accurate blade motion and loading information provided as input. In this paper, analytic formulations for the pressure gradient are derived starting with formulation 1A. Basing the new pressure gra- 
dient formulations on formulation $1 \mathrm{~A}$ has several distinct advantages. First, no additional input data is needed to predict the acoustic pressure gradient beyond what is already required to predict thickness and loading noise (or at most, numerical differentiation of the input data). Second, the retarded time algorithms that will be used have been refined and thoroughly tested in various numerical implementations of formulation 1A. Finally, by analytically computing the acoustic pressure gradient, rather than using a purely numerical approach, significant computation savings (in terms of run time and memory) and increased robustness are expected. Furthermore, the computation of the acoustic pressure from the isolated rotor can be computed concurrently with the acoustic pressure gradient.

The goal of this paper is to derive two acoustic pressure gradient formulations, and validate them through a comparison with the finite difference approximation to the acoustic pressure gradient. The formulations have been implemented in the rotor noise prediction code, PSU-WOPWOP. This code will also be used to investigate the efficiency of the formulations. It will be demonstrated that these new formulations yield accurate and efficient predictions of the acoustic pressure gradient.

\section{Acoustic Pressure Gradient Formulations}

The acoustic pressure gradient formulation derivation starts with Farassat's Formulation $1 \mathrm{~A} ; ;^{7,8}$ therefore a brief review of this formulation will be given. The PSU-WOPWOP rotor noise prediction code ${ }^{9-11}$ is used in this work to predict the acoustic pressure of rotor noise, as well as the gradient of the acoustic pressure on the scattering body.

\section{Formulation 1A}

Farassat's Formulation $1 \mathrm{~A}^{7,8}$ is an integral representation of the solution to the FW-H equation, without the quadrupole source term. It is a retarded-time formulation, which may be written as:

$$
p^{\prime}(\mathbf{x}, t)=p_{T}^{\prime}(\mathbf{x}, t)+p_{L}^{\prime}(\mathbf{x}, t)
$$

where $p^{\prime}, p_{T}^{\prime}$, and $p_{L}^{\prime}$ denote the acoustic pressure, thickness component of acoustic pressure, and the loading component of acoustic pressure, respectively. The thickness noise contribution $p_{T}^{\prime}$ can be written:

$$
4 \pi p_{T}^{\prime}(\mathbf{x}, t)=\int_{f=0}\left[\frac{\rho_{0}\left(\dot{U}_{n}+U_{\dot{n}}\right)}{r\left(1-M_{r}\right)^{2}}\right]_{\mathrm{ret}} d S+\int_{f=0}\left[\frac{\rho_{0} U_{n}\left(r \dot{M}_{r}+c\left(M_{r}-M^{2}\right)\right)}{r^{2}\left(1-M_{r}\right)^{3}}\right]_{\mathrm{ret}} d S
$$


while the loading noise contribution $p_{L}^{\prime}$ is written as:

$$
\begin{aligned}
4 \pi p_{L}^{\prime}(\mathbf{x}, t)= & \frac{1}{c} \int_{f=0}\left[\frac{\dot{L}_{r}}{r\left(1-M_{r}\right)^{2}}\right]_{\mathrm{ret}} d S+\int_{f=0}\left[\frac{L_{r}-L_{M}}{r^{2}\left(1-M_{r}\right)^{2}}\right]_{\mathrm{ret}} d S \\
& +\frac{1}{c} \int_{f=0}\left[\frac{L_{r}\left(r \dot{M}_{r}+c\left(M_{r}-M^{2}\right)\right)}{r^{2}\left(1-M_{r}\right)^{3}}\right]_{\mathrm{ret}} d S
\end{aligned}
$$

where $(\mathbf{x}, t)$ and $(\mathbf{y}, \tau)$ are the observer and source space-time variables, respectively, $r=$ $|\mathbf{x}-\mathbf{y}|$ and $c$ is the speed of sound in the undisturbed medium. The blade surface is described implicitly by the equation $f(\mathbf{y}, \tau)=0$, where $f(\mathbf{y}, \tau)$ is defined in such a way that $\nabla f=\hat{\mathbf{n}}$, which is the unit outward normal to the blade surface with components $n_{i}$. The density of the undisturbed medium is $\rho_{0}$ and $\delta(f)$ is the Dirac delta function with support on the blade surface $f=0$. In equations (2) and (3) the subscripts imply the dot product the vector with either the unit vector in the radiation direction $\hat{\mathbf{r}}$, outward normal vector $\hat{\mathbf{n}}$ to the surface $f=0$, or the surface Mach number $\mathbf{M}$. The dot over a variable indicates source time differentiation. The variables $U_{i}$ and $L_{i}$ are defined:

$$
\begin{gathered}
U_{i}=\left[1-\left(\rho / \rho_{0}\right)\right] v_{i}+\left(\rho u_{i} / \rho_{0}\right) \\
L_{i}=P_{i j} \hat{n}_{j}+\rho u_{i}\left(u_{n}-v_{n}\right)
\end{gathered}
$$

where $u_{i}$ are the components of the local flow velocity vector and $v_{i}$ are the components of the local blade surface velocity vector.

Equations (4) and (5) are the form used for a permeable surface, which is useful if the flow field around the rotor blades becomes transonic — as is the case for high-speed-impulsive noise. Equations (1)-(3) omit the quadrupole term in the FW-H equation, so significant nonlinear sources should be contained within a permeable surface. This enables the inclusion of the contribution of those sources without carrying out a volume integration. For an impermeable surface, i.e., the actual blade surface, $U_{i}=v_{i}$ and $L_{i}=P_{i j} \hat{n}_{j}$.

\section{Formulation G1}

Taking the gradient of equations (2) and (3) directly involves complicated algebraic manipulations. It is easier to start with the partial differential equation form of the FW-H equation and then use the free space Green's function to derive the new integral formulation. Details of this approach can be found in reference. ${ }^{12}$ In this paper, the formulation is revisited with slightly different notation.

The acoustic pressure gradient can be found by taking the gradient of the FW-H thickness 
and loading noise terms (neglecting the quadrupole source). The gradient of equation (1) is:

$$
\nabla p^{\prime}=\nabla p_{T}^{\prime}+\nabla p_{L}^{\prime}
$$

The next step is to find the acoustic pressure gradient of the thickness and loading noise sources. The governing equation for the thickness noise is:

$$
\square^{2} p_{T}^{\prime}=\frac{\partial}{\partial t}\left[\rho_{o} U_{n} \delta(f)\right]
$$

Using the free space Green's function $\delta(g) / 4 \pi r$, where $g=\tau-t+r / c$, the thickness component of pressure can be expressed as:

$$
4 \pi p_{T}^{\prime}(\mathbf{x}, t)=\frac{\partial}{\partial t} \int_{-\infty}^{t} \int_{-\infty}^{\infty} \frac{\rho_{0} U_{n}}{r} \delta(f) \delta(g) d \mathbf{y} d \tau
$$

Taking the gradient of equation (8) yields:

$$
\begin{aligned}
4 \pi \nabla p_{T}^{\prime}(\mathbf{x}, t) & =\nabla \frac{\partial}{\partial t} \int_{-\infty}^{t} \int_{-\infty}^{\infty} \frac{\rho_{0} U_{n}}{r} \delta(f) \delta(g) d \mathbf{y} d \tau \\
& =\frac{\partial}{\partial t} \int_{-\infty}^{t} \int_{-\infty}^{\infty} \rho_{0} U_{n} \delta(f) \nabla_{x}\left(\frac{\delta(g)}{r}\right) d \mathbf{y} d \tau
\end{aligned}
$$

where the symbol $\nabla_{x}$ stands for gradient operator with respect to the observer variable $\mathbf{x}$. The spatial gradient operator can replaced by a time derivative using the relation:

$$
\nabla_{x}\left(\frac{\delta(g)}{r}\right)=-\frac{1}{c} \frac{\partial}{\partial t}\left(\frac{\hat{\mathbf{r}} \delta(g)}{r}\right)-\frac{\hat{\mathbf{r}} \delta(g)}{r^{2}}
$$

Combining equations (9) and (10) yields:

$$
4 \pi \nabla p_{T}^{\prime}(\mathbf{x}, t)=-\frac{\partial}{\partial t}\left(\frac{1}{c} \frac{\partial}{\partial t} \int_{-\infty}^{t} \int_{-\infty}^{\infty} \frac{\hat{\mathbf{r}} \rho_{0} U_{n}}{r} \delta(f) \delta(g) d \mathbf{y} d \tau+\int_{-\infty}^{t} \int_{-\infty}^{\infty} \frac{\hat{\mathbf{r}} \rho_{0} U_{n}}{r^{2}} \delta(f) \delta(g) d \mathbf{y} d \tau\right)
$$

Using generalized function theory and geometry ${ }^{13-15}$ - and following the same steps Farassat used in deriving Formulation 1A - the gradient of the thickness component of acoustic 
pressure is found to be:

$$
\begin{aligned}
4 \pi \nabla p_{T}^{\prime}(\mathbf{x}, t) & =-\frac{\partial}{\partial t}\left(\frac{1}{c} \frac{\partial}{\partial t} \int_{f=0}\left[\frac{\hat{\mathbf{r}} \rho_{0} U_{n}}{r\left(1-M_{r}\right)}\right]_{\mathrm{ret}} d S+\int_{f=0}\left[\frac{\hat{\mathbf{r}} \rho_{0} U_{n}}{r^{2}\left(1-M_{r}\right)}\right]_{\mathrm{ret}} d S\right) \\
& =-\frac{\partial E_{1}}{\partial t}
\end{aligned}
$$

By recalling that

$$
\left.\frac{\partial}{\partial t}[\ldots]\right|_{\mathbf{x}}=\left[\left.\frac{1}{1-M_{r}} \frac{\partial}{\partial \tau}[\ldots]\right|_{\mathbf{x}}\right]_{\mathrm{ret}}
$$

and

$$
\frac{\partial \hat{\mathbf{r}}}{\partial \tau}=\frac{c}{r}\left(M_{r} \hat{\mathbf{r}}-\mathbf{M}\right)
$$

it can be easily shown that

$$
E_{1}=\frac{1}{c} \int_{f=0}\left[\hat{\mathbf{r}} E_{T}\right]_{\mathrm{ret}} d S+\int_{f=0}\left[\frac{(\hat{\mathbf{r}}-\mathbf{M}) \rho_{0} U_{n}}{r^{2}\left(1-M_{r}\right)^{2}}\right]_{\mathrm{ret}} d S
$$

where

$$
E_{T}=\left[\frac{\rho_{0}\left(\dot{U}_{n}+U_{\dot{n})}\right.}{r\left(1-M_{r}\right)^{2}}\right]_{\mathrm{ret}}+\left[\frac{\rho_{0} U_{n}\left(r \dot{M}_{r}+c\left(M_{r}-M^{2}\right)\right.}{r^{2}\left(1-M_{r}\right)^{3}}\right]_{\mathrm{ret}}
$$

is the combined thickness noise integrand in Formulation 1A; hence, it is already available in the noise prediction code. Finally, the thickness component of the acoustic pressure gradient can be written:

$$
4 \pi \nabla p_{T}^{\prime}(\mathbf{x}, t)=-\frac{\partial}{\partial t}\left\{\frac{1}{c} \int_{f=0}\left[\hat{\mathbf{r}} E_{T}\right]_{\mathrm{ret}} d S+\int_{f=0}\left[\frac{(\mathbf{r}-\mathbf{M}) \rho_{0} U_{n}}{r^{2}\left(1-M_{r}\right)^{2}}\right]_{\mathrm{ret}} d S\right\}
$$

The observer time derivative of the two integrals will be determined numerically.

The derivation of the gradient of the loading noise component of acoustic pressure follows the same procedure as used in the thickness noise noise component. The governing equation for the loading noise is written as:

$$
\square^{2} p_{L}^{\prime}=-\nabla \cdot[\mathbf{L} \delta(f)]
$$


thus the loading component of acoustic pressure is:

$$
\begin{aligned}
4 \pi p_{L}^{\prime}(\mathbf{x}, t) & =-\nabla \cdot \int_{-\infty}^{t} \int_{-\infty}^{\infty} \frac{\mathbf{L}}{r} \delta(f) \delta(g) d \mathbf{y} d \tau \\
& =-\int_{-\infty}^{t} \int_{-\infty}^{\infty} \delta(f) \mathbf{L} \cdot \nabla_{x}\left(\frac{\delta(g)}{r} d \mathbf{y} d \tau\right)
\end{aligned}
$$

Using equation (10) in the previous integral yields:

$$
4 \pi p_{L}^{\prime}(\mathbf{x}, t)=\frac{1}{c} \frac{\partial}{\partial t} \int_{-\infty}^{t} \int_{-\infty}^{\infty} \frac{L_{r}}{r} \delta(f) \delta(g) d \mathbf{y} d \tau+\int_{-\infty}^{t} \int_{-\infty}^{\infty} \frac{L_{r}}{r^{2}} \delta(f) \delta(g) d \mathbf{y} d \tau
$$

Then if the gradient of the loading component of acoustic pressure is taken, the result is:

$$
4 \pi \nabla p_{L}^{\prime}(\mathbf{x}, t)=\frac{1}{c} \frac{\partial}{\partial t} \int_{-\infty}^{t} \int_{-\infty}^{\infty} \delta(f) \mathbf{L} \cdot \nabla_{x}\left(\frac{\hat{\mathbf{r}} \delta(g)}{r}\right) d \mathbf{y} d \tau+\int_{-\infty}^{t} \int_{-\infty}^{\infty} \delta(f) \mathbf{L} \cdot \nabla_{x}\left(\frac{\hat{\mathbf{r}} \delta(g)}{r^{2}}\right) d \mathbf{y} d \tau
$$

Note that the observer and the source space-time variables are independent because none of Dirac delta functions have been integrated. This approach makes it easy to interpret the differentiation operators. Had we been working with the integrated results, we would be dealing with heavy algebraic manipulations and the differentiation operators would require careful interpretation.

Using following relations:

$$
\begin{aligned}
\mathbf{L} \cdot \nabla_{x}\left(\frac{\hat{\mathbf{r}} \delta(g)}{r}\right) & =\mathbf{L} \cdot \nabla_{x}\left(\frac{\hat{\mathbf{r}}}{r}\right) \delta(g)+\frac{L_{r} \hat{\mathbf{r}}}{c r} \delta^{\prime}(g) \\
& =\frac{\mathbf{L}-2 L_{r} \hat{\mathbf{r}}}{r^{2}} \delta(g)-\frac{L_{r} \hat{\mathbf{r}}}{c r} \frac{\partial}{\partial t} \delta(g) \\
\mathbf{L} \cdot \nabla_{x}\left(\frac{\hat{\mathbf{r}} \delta(g)}{r^{2}}\right) & =\mathbf{L} \cdot \nabla_{x}\left(\frac{\hat{\mathbf{r}}}{r^{2}}\right) \delta(g)+\frac{L_{r} \hat{\mathbf{r}}}{c r^{2}} \delta^{\prime}(g) \\
& =\frac{\mathbf{L}-3 L_{r} \hat{\mathbf{r}}}{r^{3}} \delta(g)-\frac{L_{r} \hat{\mathbf{r}}}{c r^{2}} \frac{\partial}{\partial t} \delta(g)
\end{aligned}
$$


leads to:

$$
\begin{aligned}
4 \pi \nabla p_{L}^{\prime}(\mathbf{x}, t)= & \frac{1}{c} \frac{\partial}{\partial t}\left\{-\frac{1}{c} \frac{\partial}{\partial t} \int_{-\infty}^{t} \int_{-\infty}^{\infty} \frac{L_{r} \hat{\mathbf{r}}}{r} \delta(f) \delta(g) d \mathbf{y} d \tau+\int_{-\infty}^{t} \int_{-\infty}^{\infty} \frac{\left(\mathbf{L}-3 L_{r} \hat{\mathbf{r}}\right)}{r^{2}} \delta(f) \delta(g) d \mathbf{y} d \tau\right\} \\
& +\int_{-\infty}^{t} \int_{-\infty}^{\infty} \frac{\left(\mathbf{L}-3 L_{r} \hat{\mathbf{r}}\right)}{r^{3}} \delta(f) \delta(g) d \mathbf{y} d \tau
\end{aligned}
$$

Again following the procedure used for formulation 1A, equation (24) can be rewritten as:

$$
\begin{aligned}
4 \pi \nabla p_{L}^{\prime}(\mathbf{x}, t)= & \frac{1}{c} \frac{\partial}{\partial t}\left\{-\frac{1}{c} \int_{f=0}\left[\frac{1}{1-M_{r}} \frac{\partial}{\partial \tau}\left(\frac{L_{r} \hat{\mathbf{r}}}{r\left(1-M_{r}\right)}\right)\right]_{\mathrm{ret}} d S+\int_{f=0}\left[\frac{\mathbf{L}-3 L_{r} \hat{\mathbf{r}}}{r^{2}\left(1-M_{r}\right)}\right]_{\mathrm{ret}} d S\right\} \\
& +\int_{f=0}\left[\frac{\mathbf{L}-3 L_{r} \hat{\mathbf{r}}}{r^{3}\left(1-M_{r}\right)}\right]_{\mathrm{ret}} d S
\end{aligned}
$$

Simplifying equation (25) gives the gradient of the loading noise component of acoustic pressure:

$$
\begin{aligned}
4 \pi \nabla p_{L}^{\prime}(\mathbf{x}, t) & =\frac{1}{c} \frac{\partial}{\partial t}\left\{-\int_{f=0}\left[\hat{\mathbf{r}} E_{L}\right]_{\mathrm{ret}} d S+\int_{f=0}\left[\frac{\mathbf{L}-L_{r} \hat{\mathbf{r}}}{r^{2}\left(1-M_{r}\right)}\right]_{\mathrm{ret}} d S\right. \\
& \left.-\int_{f=0}\left[\frac{L_{r} \hat{\mathbf{r}}-L_{r} \mathbf{M}}{r^{2}\left(1-M_{r}\right)^{2}}\right]_{\mathrm{ret}} d S\right\}+\int_{f=0}\left[\frac{\mathbf{L}-3 L_{r} \hat{\mathbf{r}}}{r^{3}\left(1-M_{r}\right)}\right]_{\mathrm{ret}} d S
\end{aligned}
$$

where $E_{L}$ is the combined loading noise integrand in Formulation 1A:

$$
E_{L}=\frac{1}{c}\left[\frac{\dot{L}_{r}}{r\left(1-M_{r}\right)^{2}}\right]_{\mathrm{ret}}+\left[\frac{L_{r}-L_{M}}{r^{2}\left(1-M_{r}\right)^{2}}\right]_{\mathrm{ret}}+\frac{1}{c}\left[\frac{L_{r}\left(r \dot{M}_{r}+c\left(M_{r}-M^{2}\right)\right)}{r^{2}\left(1-M_{r}\right)^{3}}\right]_{\mathrm{ret}}
$$

Again, the observer time derivative in equation (26) needs to be taken numerically.

For convenience, equations (17) and (26) are together referred to as formulation G1. This notation parallels that used by Farassat for the thickness and loading Formulation 1, which had a observer time derivative outside of the integrals. Evaluation of the pressure gradient can now be completed with substantially less computational effort than a direct numerical evaluation of the pressure gradient. More details of the derivation of formulation G1 are given by Farassat and Brentner. ${ }^{12}$

Equations (17) and (26) have been implemented in the PSU-WOPWOP noise prediction 
code to provide the acoustic pressure gradient at an arbitrary observer location. The main challenge of this implementation is the calculation of observer time derivative, $\partial / \partial t$, of the integrals. Care must be taken to ensure that the observer position $\mathbf{x}$ remain fixed during the calculation of these integrals. To simplify the algorithm description, the integrals which much be differentiated, surrounded by the braces in equation (26), are represented by $\mathrm{Q}$. A second-order backward difference algorithm is used to compute the time derivative. The general algorithm for the numerical calculation of $\partial / \partial t$ is as follows:

A. Pick $\tau^{n}-n$ indicates time step and $\tau$ represents the emission or retarded time.

B. Compute $\mathbf{y}_{i}\left(\tau^{n}\right)$ - each source point is moving, thus at time $n$, the position of the $i$ source point is needed. source point.

C. Save $\tau^{n}, \mathbf{y}_{i}\left(\tau^{n}\right)$, velocity, acceleration, etc. for later use as the $\tau^{n-1}$ and $\tau^{n-2}$ values once $n$ has been incremented.

D. Compute $\mathbf{x}\left(t^{n}\right)$ (based on $\mathbf{y}_{i}\left(\tau^{n}\right)$ and $\tau^{n}$ ) - if the source location is fixed, $\mathbf{x}$ does not change, and the arrival time $t$ is found by $t=\tau+r / c$; if the observer is moving, both the observer position and arrival (observer) time must be determined.

E. Calculate $Q\left(\mathbf{y}_{i}, \tau^{n} ; \mathbf{x}\left(t^{n}\right), t_{x^{n}}^{n}\right) \equiv Q_{n}^{n}$ using velocity, acceleration etc. at $\tau^{n}$

F. Compute $t_{x^{n}}^{n-1}$ and $t_{x^{n}}^{n-2}$ using $\tau^{n-1}$ and $\tau^{n-2}$ as follows:

i. $\quad t_{x^{n}}^{n-1}=\tau^{n-1}+\left|\mathbf{x}\left(t^{n}\right)-\mathbf{y}_{i}\left(\tau^{n-1}\right)\right| / c$

ii. $t_{x^{n}}^{n-2}=\tau^{n-2}+\left|\mathbf{x}\left(t^{n}\right)-\mathbf{y}_{i}\left(\tau^{n-2}\right)\right| / c$

G. Calculate $Q\left(\mathbf{y}_{i}^{n-1}, \tau^{n-1} ; \mathbf{x}\left(t^{n}\right), t_{x^{n}}^{n-1}\right) \equiv Q_{n}^{n-1}$ and $Q\left(\mathbf{y}_{i}^{n-2}, \tau^{n-2} ; \mathbf{x}\left(t^{n}\right), t_{x^{n}}^{n-2}\right) \equiv Q_{n}^{n-2}$

H. Calculate $\frac{\partial Q}{\partial t} \cong \frac{Q_{n}^{n-2}-(1+\alpha)^{2} Q_{n}^{n-1}+\alpha(\alpha+2) Q_{n}^{n}}{\alpha(1+\alpha)\left(t_{n}^{n}-t_{n}^{n-1}\right)}$ where $\alpha=\frac{t_{n}^{n-1}-t_{n}^{n-2}}{t_{n}^{n}-t_{n}^{n-1}}$ for a non-uniform time step

I. Interpolate $\frac{\partial Q}{\partial t}$ at $t^{*}$

where $\tau$ denotes source time, $t$ observer time, $y_{i}$ source vector, $x_{i}$ observer vector, $c$ speed of sound, $n$ time index and $t^{*}$ is the specified observer time of interest. It is apparent that this procedure is significantly more complicated than computing the acoustic pressure. Nevertheless, the additional computational effort will be shown to be significantly less than a purely numerical differentiation of the acoustic pressure. 


\section{Formulation G1A}

The primary drawback of Formulation G1 is that numerical time differentiation of the integrals is required. If the observer is stationary, then this requirement is not a problem because the time history of the integrals can be easily differentiated numerically. If the observer is moving with respect to the fluid, as in the case of a wind-tunnel test, the situation becomes more complicated because the formulation requires the observer to be stationary during the evaluation of the integrals. Predictions with a moving observer are possible by adjusting the observer position at each time in the acoustic-pressure time history; however, three evaluation of the integrals are needed to perform a second-order difference approximation to the time derivatives at each observer time. These extra integral evaluations become unnecessary if the time derivatives are taken inside the integrals analytically.

Although the process of taking the observer time derivatives inside the integrals and converting them to source time derivatives is not difficult, it is quite tedious. The first step is to apply equation (13) and then evaluate the source time derivatives that results. Some of the key source time derivatives, which are the same as Farassat used in the derivation of Formulation 1A, are expressed as follows:

$$
\begin{gathered}
\frac{\partial \hat{\mathbf{r}}}{\partial \tau}=\frac{c}{r}\left(M_{r} \hat{\mathbf{r}}-\mathbf{M}\right) \\
\frac{\partial r}{\partial \tau}=-c M_{r} \\
\frac{\partial}{\partial \tau}\left(\frac{1}{r}\right)=-\frac{1}{r^{2}} \frac{\partial r}{\partial \tau}=\frac{c M_{r}}{r^{2}} \\
\frac{\partial M_{r}}{\partial \tau}=\frac{c}{r}\left(-M^{2}+M_{r}^{2}\right)+\dot{M}_{r}
\end{gathered}
$$

Some new functions are introduced denoted by the following symbols:

$$
\begin{gathered}
W=r \dot{M}_{r}+c\left(M_{r}-M^{2}\right) \\
\dot{W}=\frac{r^{2} \ddot{M}_{r}-3 c r \dot{\mathbf{M}} \cdot \mathbf{M}+c\left(r \dot{M}_{r}+c\left(M_{r}^{2}-M^{2}\right)\right)}{r} \\
U(m, n)=\frac{1}{r^{m}\left(1-M_{r}\right)^{n}} \\
V(m, n)=\frac{\partial U(m, n)}{\partial \tau}=\frac{n r \dot{M}_{r}+(n-m) c M_{r}^{2}+m c M_{r}-n c M^{2}}{r^{m+1}\left(1-M_{r}\right)^{n+1}}
\end{gathered}
$$

or

$$
V(m, n)=n W U(m+1, n+1)+c(m-n) M_{r} U(m+1, n)
$$


These relations will not be used in the process of taking the observer time derivatives inside the integrals in Formulation G1.

Equations (17) and (26) are the starting point for the derivation of Formulation G1A. They can be written in a short hand notation for convenience:

$$
\begin{gathered}
4 \pi \nabla p_{T}^{\prime}(\mathbf{x}, t)=I_{1}+I_{2} \\
4 \pi \nabla p_{L}^{\prime}(\mathbf{x}, t)=I_{3}+I_{4}+I_{5}+I_{6}
\end{gathered}
$$

where each of individual integrals $I_{1}-I_{6}$ are given below. In these descriptions, it is helpful to define $\rho_{0} U_{n}$ as $Q$, then

$$
\begin{aligned}
& I_{1}=-\frac{1}{c} \int_{f=0}\left[\frac { 1 } { 1 - M _ { r } } \left\{\frac{c\left(M_{r} \hat{\mathbf{r}}-\mathbf{M}\right)}{r}(\dot{Q} U(1,2)+Q W U(2,3))\right.\right. \\
& +\hat{\mathbf{r}}\{\ddot{Q} U(1,2)+\dot{Q}(V(1,2)+W U(2,3)) \\
& +Q(\dot{W} U(2,3)+W V(2,3))\}\}]_{\text {ret }} d S \\
& I_{2}=\int_{f=0}\left[\frac { 1 } { 1 - M _ { r } } \left\{\left(\frac{r \dot{\mathbf{M}}-c M_{r} \hat{\mathbf{r}}+c \mathbf{M}}{r}\right) Q U(2,2)\right.\right. \\
& -(\hat{\mathbf{r}}-\mathbf{M})(\dot{Q} U(2,2)+Q V(2,2))\}]_{\mathrm{ret}} d S \\
& I_{3}=-\frac{1}{c^{2}} \int_{f=0} \frac{1}{1-M_{r}}\left[\frac{\partial \hat{\mathbf{r}}}{\partial \tau}\left\{\dot{L}_{r} U(1,2)+c\left(L_{r}-L_{M}\right) U(2,2)+L_{r} W U(2,3)\right\}\right. \\
& +\hat{\mathbf{r}}\left\{\ddot{L}_{r} U(1,2)+\dot{L}_{\dot{r}} U(1,2)+\dot{L}_{r} V(1,2)\right. \\
& \left.+c\left(\frac{\partial L_{r}}{\partial \tau}-\dot{L}_{M}-L_{\dot{M}}\right) U(2,2)+c\left(L_{r}-L_{M}\right) V(2,2)\right\} \\
& \left.+\frac{\partial L_{r}}{\partial \tau} W U(2,3)+L_{r} \dot{W} U(2,3)+L_{r} W V(2,3)\right]_{\mathrm{ret}} d S \\
& I_{4}=\frac{1}{c} \int_{f=0} \frac{1}{1-M_{r}}\left[\left(\dot{\mathbf{L}}-\frac{\partial L_{r}}{\partial \tau} \hat{\mathbf{r}}-L_{r} \frac{\partial \hat{\mathbf{r}}}{\partial \tau}\right) U(2,1)+\left(\mathbf{L}-L_{r} \hat{\mathbf{r}}\right) V(2,1)\right]_{\mathrm{ret}} d S
\end{aligned}
$$




$$
\begin{gathered}
I_{5}=-\frac{1}{c} \int_{f=0} \frac{1}{1-M_{r}}\left[\frac{\partial L_{r}}{\partial \tau}(\hat{\mathbf{r}}-\mathbf{M}) U(2,2)+L_{r}\left(\frac{\partial \hat{\mathbf{r}}}{\partial \tau}-\dot{\mathbf{M}}\right) U(2,2)\right. \\
\left.+L_{r}(\hat{\mathbf{r}}-\mathbf{M}) V(2,2)\right]_{\mathrm{ret}} d S \\
I_{6}=\int_{f=0}\left[\frac{\mathbf{L}-3 L_{r} \hat{\mathbf{r}}}{r^{3}\left(1-M_{r}\right)}\right]_{\mathrm{ret}} d S
\end{gathered}
$$

where

$$
\frac{\partial L_{r}}{\partial \tau}=\dot{L}_{r}+\mathbf{L} \cdot \frac{\partial \hat{\mathbf{r}}}{\partial \tau}
$$

Recall that for an impermeable surface,

$$
\begin{aligned}
& Q=\rho_{0} v_{n} \\
& L=P_{i j} \hat{n}_{j}
\end{aligned}
$$

and for a permeable surface,

$$
\begin{gathered}
Q=\rho_{0} v_{n}+\rho\left(u_{n}-v_{n}\right) \\
L=P_{i j} \hat{n}_{j}+\rho u_{i}\left(u_{n}-v_{n}\right)
\end{gathered}
$$

Also remember that a dot on the main variables does not imply differentiation of any of the associated vectors implied by the subscripts. Subscripts other than $i$ and $j$ are a shorthand for the inner product of the main quantity with the vector represented by the subscript. The derivative of acceleration, which is called a jerk, and second derivative of normal unit vector were evaluated numerically in this work.

Equations (37) and (38), together with the definitions of $I_{1}-I_{6}$, will be referred to as formulation G1A and are the main result of this paper. The designation G1A is intended to parallel that of Farassat's formulation 1A, in which the observer time derivative is taken analytically inside the thickness and loading integrals. Formulation G1A does not require numerical time differentiation of the integrals and, as a retarded-time formulation, is well suited for subsonic source motion. Aside from the problem geometry, only the time-dependent input values or at most, numerical differentiation of them are required. Furthermore, it will be demonstrated with numerical examples that formulation G1A requires significantly less operations and computer memory than formulation G1. This will be discussed in detail later. The reduction of computational cost is important when the formulation is used for the scattering problem. 


\section{Results and Discussions}

In this section, two representative calculations are performed to demonstrate the capability of the new formulations and to provide some indication of the efficiency and robustness of the formulations. The first case considers mode-scale UH-1H rotor with untwisted blades operating in a non-lifting hover condition. This test enables simple and fast calculation for both the pressure and pressure gradient. The other test case is for the HART-I model rotor in a forward descent flight, which experiences blade-vortex-interaction (BVI) high frequency loading on the blades (although the CFD solution does not fully capture the BVI). Measured data is not available for the pressure gradient; therefore, the predicted pressure-gradient time histories predicted by the analytical formulations will be compared with a purely numerical approach. The finite difference predictions are performed by computing the acoustic pressure at several points nearby the observer location and then using a second-order central finite difference in each of the three spatial directions.

\section{Test case 1 : UH-1H model rotor}

A model scale rotor test, conducted by Boxwell ${ }^{16}$ et al. in 1978 and later repeated by Purcell $^{17}$ in 1988, was selected for the validation of the present theory and code. The rotor was a one-seventh scale model of a UH-1H main rotor with straight, untwisted blades. The model rotor utilized an NACA 0012 airfoil section. The rotor radius $R$ was $1.045 \mathrm{~m}$ with a chord of $7.62 \mathrm{~cm}$. The model was run at several high-speed hover conditions with low thrust. The high-speed hover condition is not of particular interest for the validation of the pressure gradient; therefore, a tip Mach number of 0.6 was selected for the test case. For the hover noise calculation, an Euler solution reported by Baeder et al. ${ }^{18}$ was used as input data. The Euler calculation were performed on a $\mathrm{C}-\mathrm{H}$ grid; only the lower half of the grid was used in the CFD calculations by taking advantage of the symmetry of the problem. The Euler calculations required approximately $80 \mathrm{~min}$ of CPU time on a Cray Y-MP. Details of the Euler calculations can be found in references.$^{18,19}$

Comparisons of the pressure gradient are made for an in-plane microphone located 3.09R from the rotor hub for a stationary observer. Figure 1 shows the total acoustic pressure and the pressure gradient with respect to $x, y$, and $z$ directions, respectively. Pressure gradient predicted by two analytical formulations are compared to that obtained by the finite difference method, which is a purely numerical approach. The agreement between analytic formulations and the finite difference method is excellent for all components of the pressure gradient. A closer examination reveals that the analytical formulations provide much smoother results as compared to the finite difference method. 
(a)

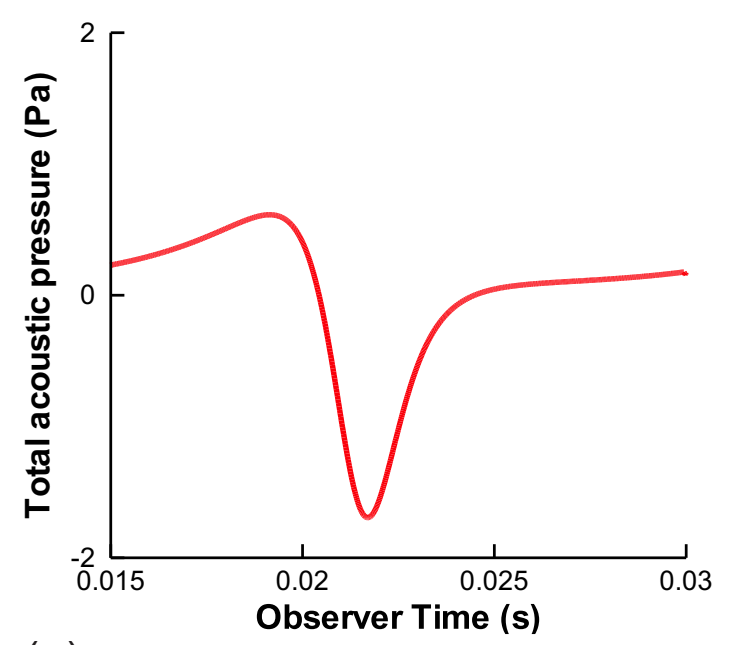

(c)

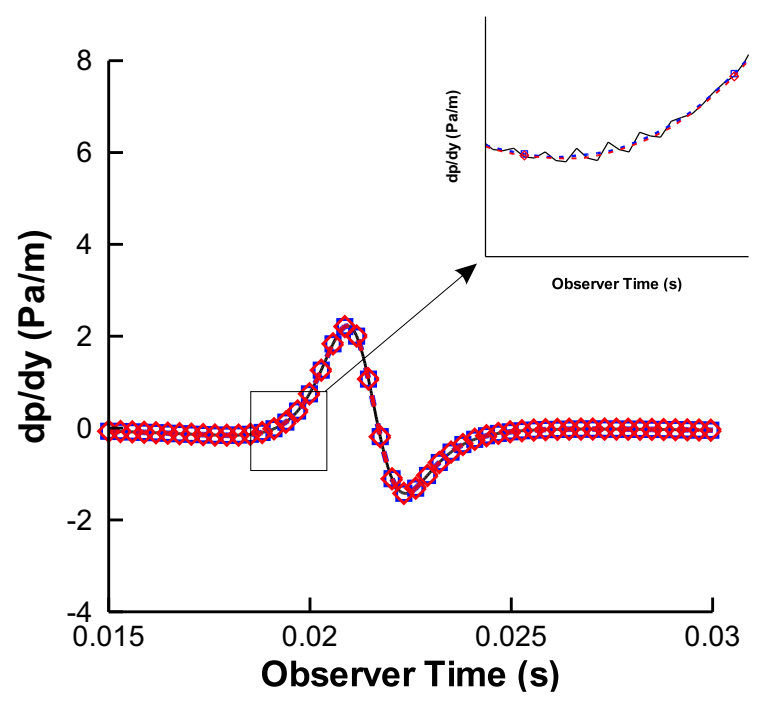

(b)

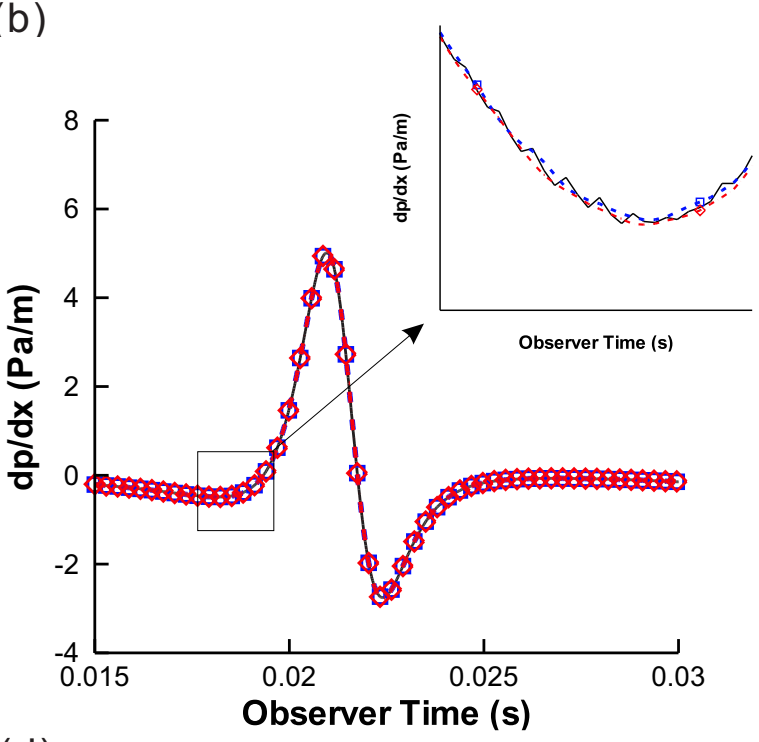

(d)

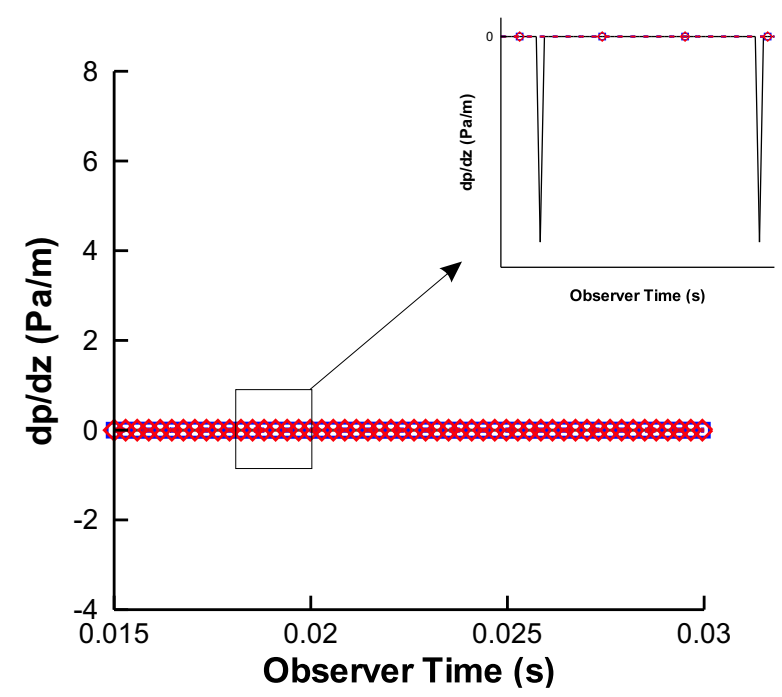

Figure 1. Acoustic pressure and the components of the acoustic pressure gradient for the UH$1 \mathbf{H}$ model rotor operating in hover with $M_{\mathrm{tip}}=0.6$. (a) total acoustic pressure (b) $\partial p^{\prime} / \partial x$ (c) $\partial p^{\prime} / \partial y$ (d) $\partial p^{\prime} / \partial z$; finite difference method: - ; formulation G1A: $--\square--$; formulation G1: - - ---- .

\section{Test Case 2: HART-I model rotor}

The forward-flight capability of the new formulations and code was demonstrated for a fourbladed rotor representative of the HART-I model-scale test. This case focuses on unsteady blade loading and forward flight. The OVERFLOW CFD code was used to compute the unsteady flow field around the rotor. ${ }^{20,21}$ A C-mesh topology was been used for the grid with a total grid system of 2.4 million points in the near-body region and 15.0 million points in the off-body region. The turbulence model used the shear stress transport (SST) ${ }^{22} k-\omega$ 


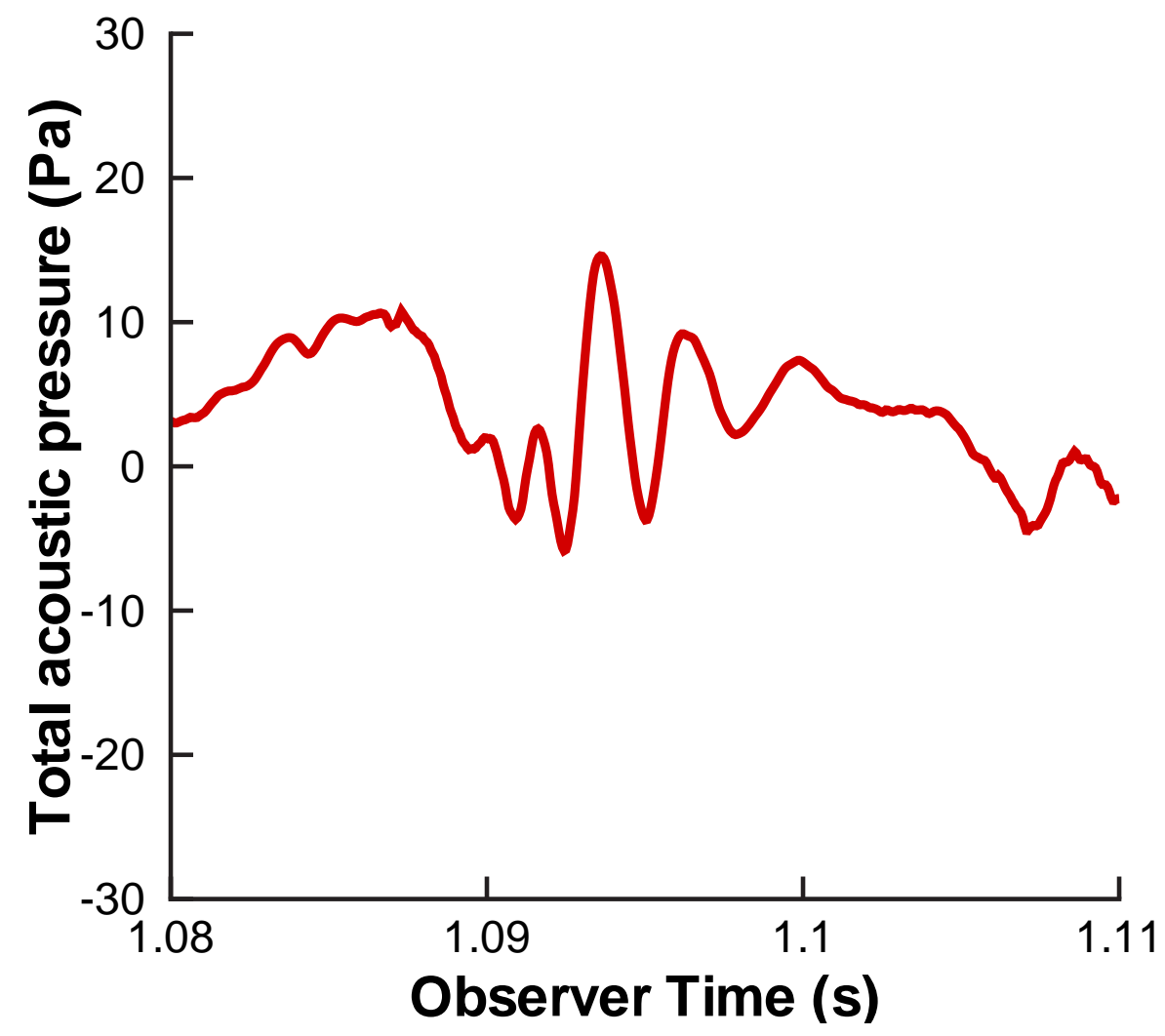

Figure 2. Acoustic pressure for the HART-I rotor operating in a BVI flight condition.

by Menter. The rapid dissipation of blade-vortex strength makes the prediction of computational fluid dynamics(CFD) difficult. Although the CFD was not fully able to capture the BVI loading on the blades - and hence the peaks of predicted noise were considerably underpredicted as shown in the reference ${ }^{20,21}$ - the comparison of the new analytical formulations for pressure gradient with the finite difference method is still useful to demonstrate the implementation in PSU-WOPWOP.

Like UH-1H examples, the result of the finite difference method was compared to that of analytic formulations to validate the newly developed formulations. For this comparison, a microphone located below the rotor plane at a downstream position on the retreating side of the rotor. The observer is in motion with the rotor to simulate the wind-tunnel test. Permeable acoustic data surfaces, which surround each of the four rotor blades, are used for the noise and pressure gradient computations. Although the absolute magnitude of the pressure gradient is unknown, confidence in both the derivation and implementation of the new formulations is gained if all of the different methods agree reasonably well.

Figure 2-5 shows the total acoustic pressure and a comparison of the pressure gradient at 


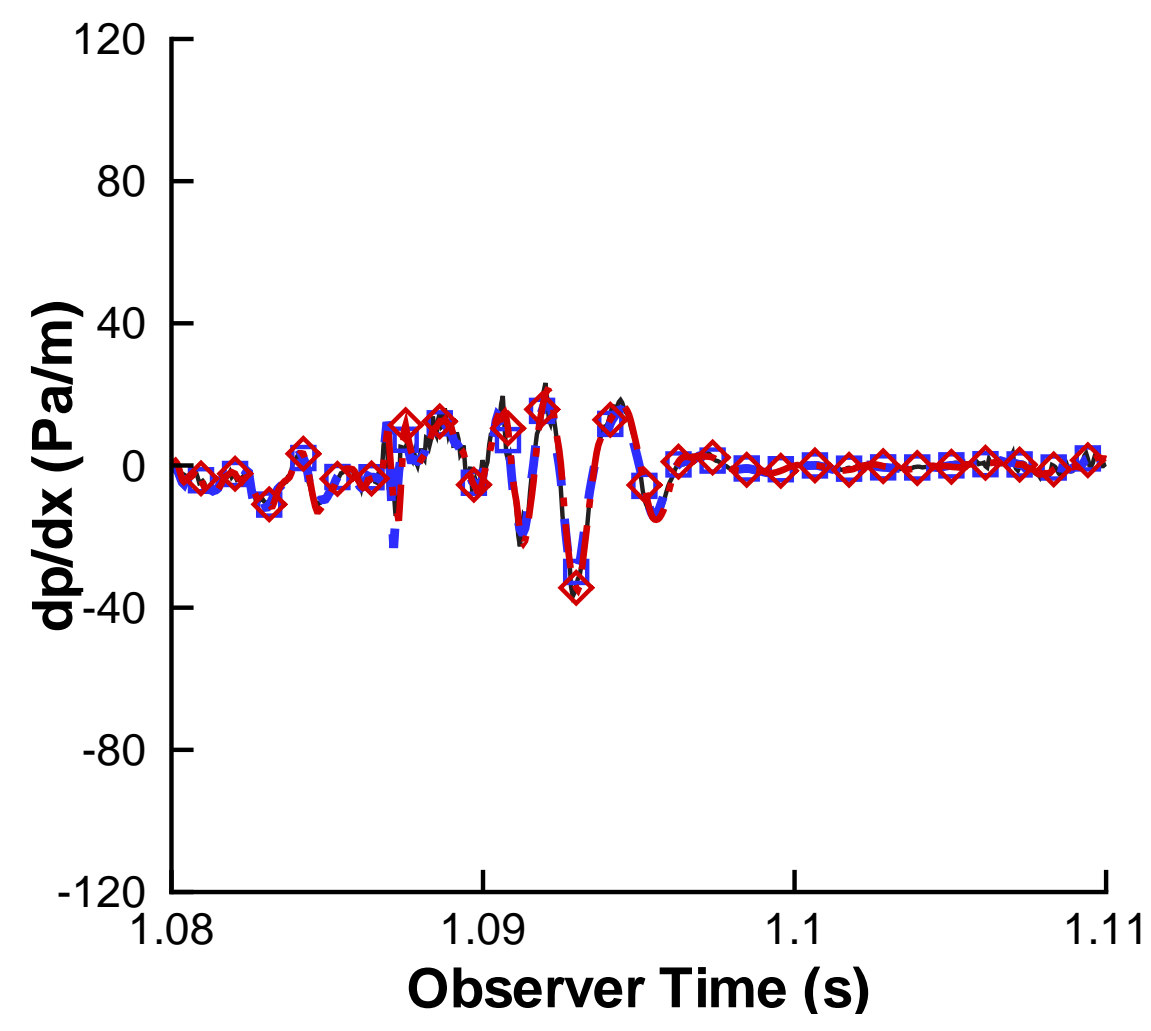

Figure 3. $x$ component of the acoustic pressure gradient $\left(\partial p^{\prime} / \partial x\right)$ for the HART-I rotor operating in a BVI flight condition.; finite difference method: $\square$; formulation G1A: $--\square--$ ; formulation G1: - - ---- .

a moving observer for the HART-I rotor. The analytical formulations are in a good agreement with the finite difference method. Upon closer inspection (not shown), the finite difference contains a high frequency "jitter" that is thought to be of numerical origin. The analytical formulations do not exhibit the same "jitter." In some other cases with at moving observer (not shown) it was found that the acoustic pressure gradient predicted by formulation G1 was sensitive to the method of computing the observer time and position. This has not been studied extensively because Formulation G1A does not suffer in this regard, and requires less computational effort.

Table 1 shows a comparison of computational time for Formulation 1A (as a reference), Formulations G1A and G1, and the finite difference method. The finite difference method required 7 times as much time as Formulation 1A but Formulation G1A only required 3 times as much computation time as Formulation 1A. Formulation G1 required approximately 5 times much computation time as Formulation 1A or 60 percent more computation time than Formulation G1A. This demonstrates the significant computational savings of both of the 


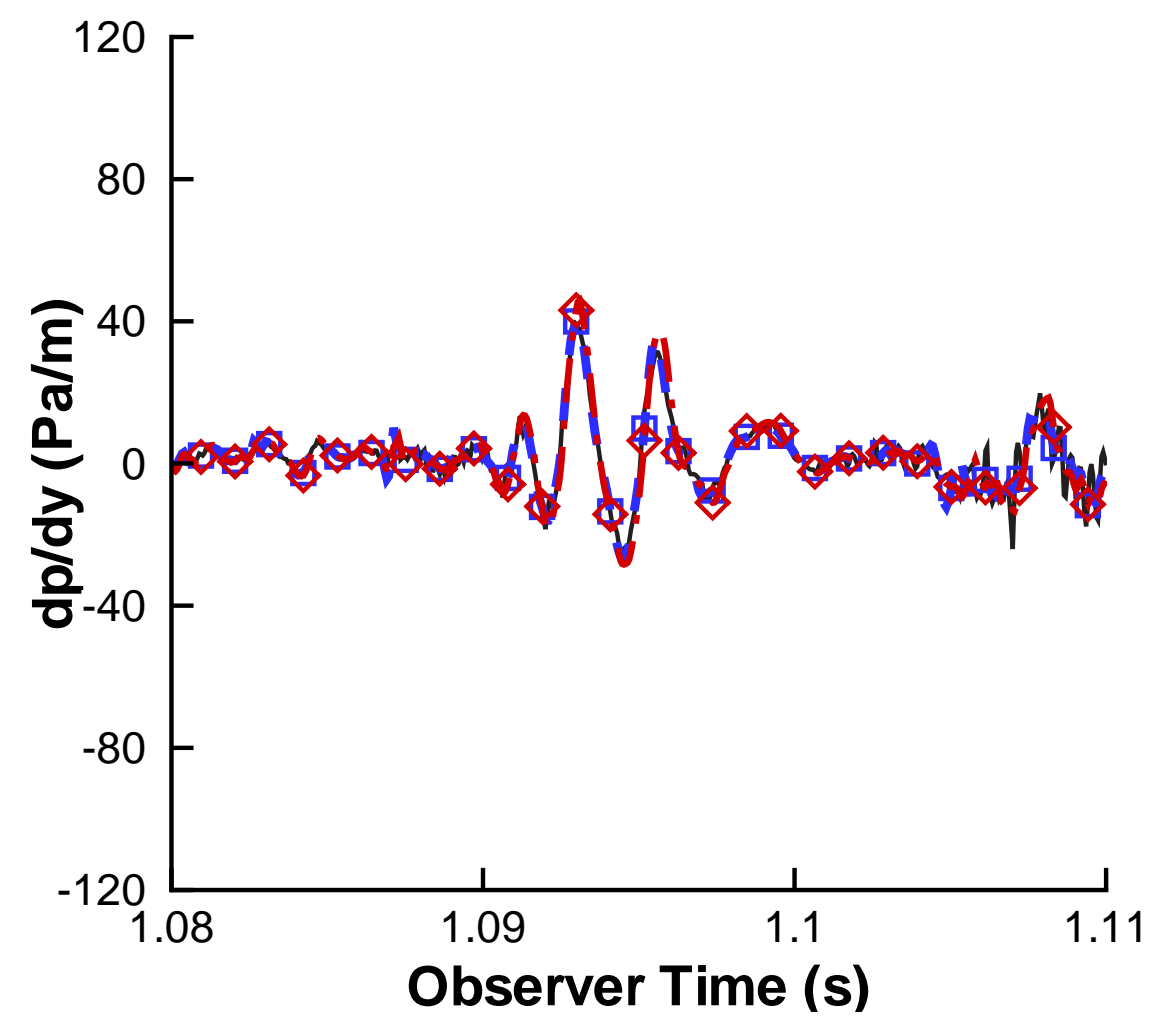

Figure 4. $y$ component of the acoustic pressure gradient $\left(\partial p^{\prime} / \partial y\right)$ for the HART-I rotor operating in a BVI flight condition.; finite difference method: - ; formulation G1A: $--\square--$ ; formulation G1: - - $\diamond---$.

analytical formulations and the superiority of Formulation G1A.

\begin{tabular}{|c|c|c|c|}
\hline Formulation 1A & Formulation G1A & Formulation G1 & Finite Difference Method \\
\hline $11.5(\mathrm{~s})$ & $31.7(\mathrm{~s})$ & $49.4(\mathrm{~s})$ & $79.0(\mathrm{~s})$ \\
\hline
\end{tabular}

Table 1. Comparison of computational time for the HART rotor with permeable surface

\section{Concluding Remarks}

In this paper, two analytical formulations for the acoustic pressure gradient of rotor noise were developed and validated by comparison with a purely numerical method for both hovering and forward-flight conditions. The analytical formulations eliminated numerical oscillations,which were present in the numerical method, and resulted in a very smooth predictions. The fact that all three approaches gave essentially the same results - although 


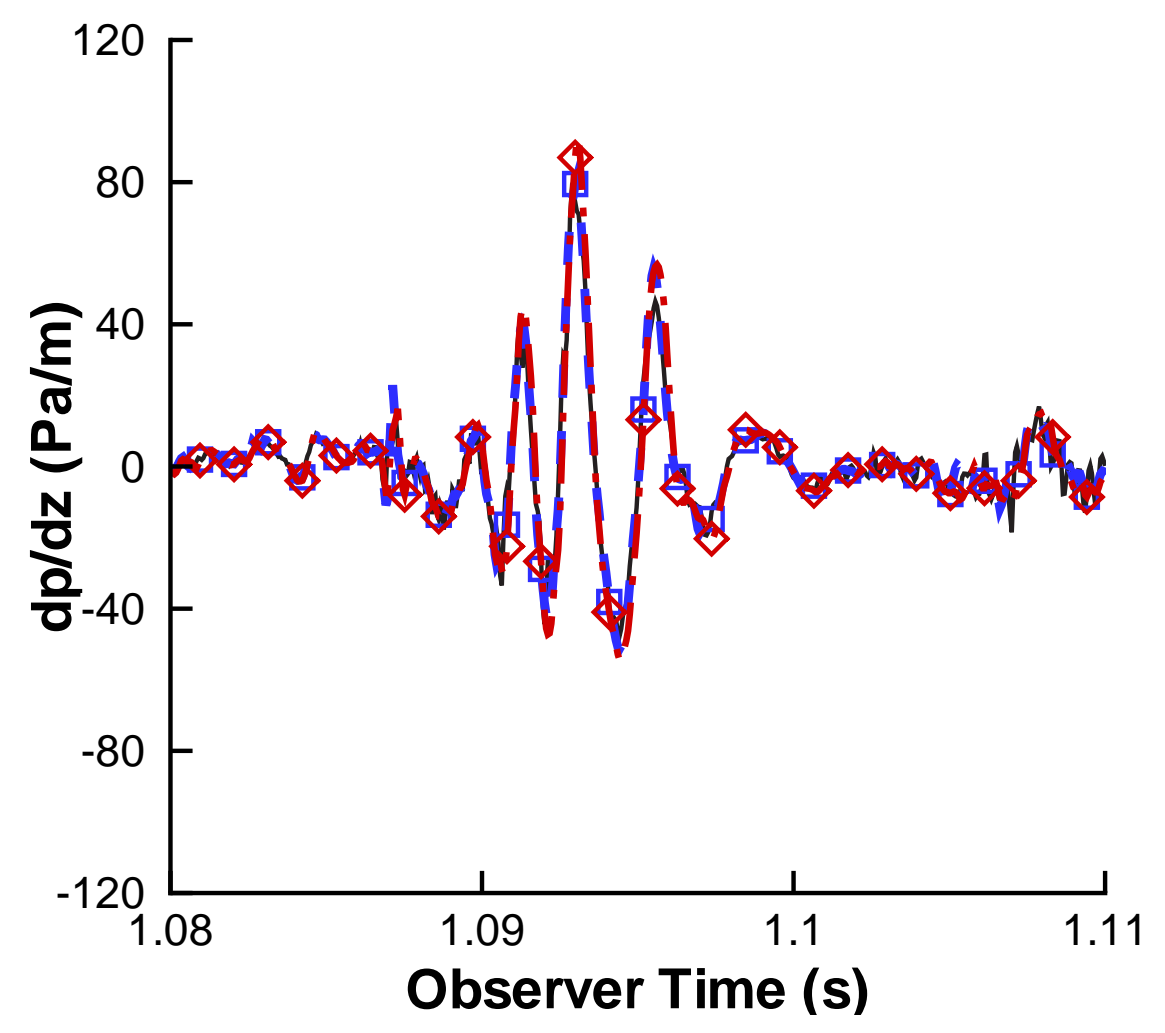

Figure 5. $z$ component of the acoustic pressure gradient $\left(\partial p^{\prime} / \partial z\right)$ for the HART-I rotor operating in a BVI flight condition.; finite difference method: - ; formulation G1A: $--\square--$ ; formulation G1: - - ---- .

they are quite different in expression and implementation - gives confidence that both the derivation and implementation have been performed correctly.

It was found that formulation G1, which evaluates the observer time differentiation of the integrals, is a relatively simple formulation but is somewhat more difficult to implement in PSU-WOPWOP due to the observer time differentiation of the acoustic integrals. Furthermore, it was sensitive to the choice of numerical algorithm used to find the observer time and location (in at least one case - not shown). In contrast, Formulation G1A, which takes the time derivatives inside the integrals, is a somewhat more complicated formulation, nevertheless, it yields improved computational efficiency and perhaps robustness by avoiding the numerical time differentiation of the acoustic integrals. Numerical tests show that Formulation G1A is the fastest and the most efficient algorithm for computing the acoustic pressure gradient. This is important for use in computing the acoustic scattering, which may require several thousand pressure gradient calculations at the collocation points on the scattering body. 


\section{Acknowledgments}

This study was conducted under the project "Prediction of Acoustic Scattering and Nonlinear Propagation for Heavy Lift Rotorcraft" of Vertical Lift Research of Excellence(VLRCOE) at Penn Sate and supported by the NASA Langley Research Center under "Maneuver and High-Speed Rotor Acoustic Analysis Development", NASA Purchase Order NNL05AD50P.

\section{References}

${ }^{1}$ Atalla, N. and Glegg, S., "A Ray-Acoustics Approach to Fuselage Scattering of Rotor Noise," AIAA Paper 90-4013, AIAA $13^{\text {th }}$ Aeroacoustics Conference, Tallahassee, FL, Oct 1990.

${ }^{2}$ Atalla, N. and Glegg, S., "Ray-Acoustics Approach to Fuselage Scattering of Rotor Noise," Journal of the American Helicopter Society, Vol. 38, No. 3, July 1993, pp. 56-63.

${ }^{3}$ Dunn, M. H. and Tinetti, A. F., "Aeroacoustic Scattering Via The Equivalent Source Method," AIAA Paper 2004-2937, AIAA $10^{\text {th }}$ AIAA/CEAS Aeroacoustics Conference, Manchester, United Kingdom, May 2004.

${ }^{4}$ Myers, M. K. and Hausmann, J. S., "Computation of acoustic scattering from a moving rigid surface," Journal of the Acoustical Society of America, Vol. 91, No. 5, May 1992, pp. 2594-2605.

${ }^{5}$ Jeon, W. and Lee, D., "A numerical study on the flow and sound fields of centrifugal impeller located near a wedge," Journal of Sound and Vibration, Vol. 266, No. 4, 2003, pp. 785-804.

${ }^{6}$ Ffowcs Williams, J. E. and Hawkings, D. L., "Sound Generated by Turbulence and Surfaces in Arbitrary Motion," Philosophical Transactions of the Royal Society, Vol. A264, No. 1151, 1969, pp. 321-342.

${ }^{7}$ Brentner, K. S., "Prediction of Helicopter Discrete Frequency Rotor Noise - A Computer Program Incorporating Realistic Blade Motions and Advanced Acoustic Formulation," NASA TM 87721, 1986.

${ }^{8}$ Farassat, F., "Derivation of Formulations 1 and 1A of Farassat," NASA TM 2007-214853, 2007.

${ }^{9}$ Brès, G. A., Modeling the noise of arbitrary maneuvering rotorcraft: Analysis and implementation of the PSU-WOPWOP noise prediction code, M.S. thesis, Department of Aerospace Engineering, The Pennsylvania State University, June 2002.

${ }^{10}$ Perez, G., Investigation of the influence of maneuver on rotorcraft noise, M.S. thesis, Department of Aerospace Engineering, The Pennsylvania State University, June, 2002.

${ }^{11}$ Brentner, K. S., Perez, G., Brès, G. A., and Jones, H. E., "Maneuvering Rotorcraft Noise Prediction," Journal of Sound and Vibration, Vol. 39, No. 3-5, August 2003, pp. 719-738.

${ }^{12}$ Farassat, F. and Brentner, K. S., "The Derivation of the Gradient of the Acoustic Pressure on a Moving Surface for Application to the Fast Scattering Code (FSC)," Tech. Rep. TM 2005-213777, NASA, July 2005.

${ }^{13}$ Farassat, F., "Introduction to Generalized Functions With Applications in Aerodynamics and Aeroacoustics," NASA TP 3428, 1994, See corrected version, April 1996. Available online at NTRS (NASA Technical Report Server).

${ }^{14}$ Gel'fand, I. M. and Shilov, G. E., Generalized Functions: Properties and Operations, Vol. 1, Academic Press, Inc., 11 Fifth Avenue, New York 3, New York, 1964, Translated by Eugene Saletan, Department of Physics, Northeastern University, Boston, Massachuesetts.

${ }^{15}$ Kanwal, R. P., Generalized Functions - Theory and Applications, Birkhauser, Boston, 3rd ed., 2004. 
${ }^{16}$ Boxwell, D. A., Yu, Y. H., and Schmitz, F. H., "Hovering Impulsive Noise: Some Measured and Calculated Results," Vertica, Vol. 3, No. 1, 1979, pp. 35-45, Also in NASA CP-2052, 1978.

${ }^{17}$ Purcell, T. W., "CFD and Transonic Helicopter Sound," Fourteenth European Rotorcraft Forum, 1988, Paper 2.

${ }^{18}$ Baeder, J. D., Gallman, J. M., and Yu, Y. H., "A Computational Study of Aeroacoustics of Rotors in Hover," American Helicopter Society 49th Annual Forum, 1993, See also. ${ }^{23}$

${ }^{19}$ Baeder, J. D., "Euler Solutions to Nonlinear Acoustics of Non-Lifting Rotor Blades," American Helicopter Society and Royal Aeronautical Society International Technical Specialists Meeting: Rotorcraft Acoustics and Rotor Fluid Dynamics, 1991.

${ }^{20}$ Makinen, S. M., Hill, M., Gandhi, F., and Long, L. N., "A Study of Higher Harmonic Airloads for Helicopter Rotors in Descent Flight with Computational Fluid Dynamics," 62th AHS Forum of the American Helicopter Society, Phoenix, Arizona, May 9-11 2006.

${ }^{21}$ Lee, S., Brentner, K. S., Hennes, C. C., Flynt, B. T., Theron, J. N., and Duque, E. P. N., "Investigation of the Accuracy Requirement for Permeable Surfaces Used in Rotor Noise Prediction," 62th AHS Forum of the American Helicopter Society, Phoenix, Arizona, May 9-11 2006.

${ }^{22}$ Menter, F. R. and Rumsey, C. L., "Assessment of Two-Equation Turbulence Models for Transonic Flows," AIAA Paper 94-2343, 1994.

${ }^{23}$ Baeder, J. D., Gallman, J. M., and Yu, Y. H., "A Computational Study of Aeroacoustics of Rotors in Hover," Journal of the American Helicopter Society, Vol. 42, No. 1, 1997, pp. 39-53. 